\title{
Las partes interesadas -stakeholders- en el proceso de desarrollo de las actividades turísticas
}

\section{Stakeholders in the tourism development process}

María Elena Heredia Mendoza*, Isabel Zizaldra Hernández ${ }^{* *}$ y Jesús Alberto Urrutia de la Garza***

\begin{abstract}
En este artículo el objetivo es ampliar la base de investigación al conceptualizar cómo se pueden gestionar los múltiples intereses de los interesados en los procesos de desarrollo y planeación del turismo. En este sentido, es significativo el surgimiento de la Teoría de las Partes Interesadas o Stakeholders para llenar el vacío de conocimiento en cuanto a la explicación de la manera en que las diferentes partes involucrados (Comunidades Locales, Turistas, Gobiernos Estatal y Federal), deben participar en la planeación del desarrollo turístico de las comunidades anfitrionas y de los beneficios obtenidos y repartidos con base en su nivel de participación. En el desarrollo de la investigación la Teoría de las Partes Interesadas se ha integrado en la elaboración de la planeación del desarrollo turístico, pues éste desarrollo ha estado influido por diversos grupos o personas con intereses diferentes y, por consiguiente, se explica la manera en que éstas diferentes partes interesadas deben estar trabajando conjuntamente en la formulación de políticas y planeación del desarrollo turístico, el trabajo conjunto genera beneficios en el desarrollo turístico de los destinos anfitriones. Lo relevante del involucramiento de las partes interesadas es la experiencia y aprendizaje en la gestión del turismo y planeación del turismo, han desempeñado, como consecuencia, un papel significativo en el desarrollo de los destinos turísticos.
\end{abstract}

Abstract

In this article, the objective is to broaden the research base by conceptualizing how the multiple interests of those interested in tourism development and planning processes can be managed. In this sense, the emergence of the Theory of Stakeholders or Stakeholders is significant in order to fill the knowledge gap regarding the explanation of how the different parties involved (Local Communities, Tourists, State and Federal Governments), must Participate in the planning of the tourism development of the host communities and of the benefits obtained and distributed based on their level of participation. In the development of research, the Theory of Interested Parties has been integrated into the development of tourism development planning, since this development has been influenced by various groups or people with different interests and, therefore, explains the way in which These different stakeholders must be working together in the formulation of policies and planning of tourism development, the joint work generates benefits in the tourism development of the host destinations. The relevant part of the involvement of interested parties is the experience and learning in tourism management and tourism planning, as a consequence, they have played a significant role in the development of tourist destinations.

\section{Introducción}

Con base en la complejidad de los negocios de hoy en día, se presenta un hueco en el conocimiento para explicar las razones por las cuales se presentan ineficiencias en el desarrollo de las actividades turísticas (Planeación del Desarrollo) en cuanto a la desigual distribución de los beneficios generados entre los diferentes agentes económicos involucrados, por lo que no permitía generar una explicación razonable en los

* Doctora en Ciencias Administrativas por la Universidad Autónoma de Ciudad Juárez.Contacto: elenita_heredia@hotmail.com. ORCID:0000-O002-82577983

** Doctora en Turismo, Administración y Derecho. Profesora investigadora de la Universidad Autónoma de Ciudad Juárez. Contacto: izizaldr@uacj.mx. ORCID: 0000-0001-7079-5521

*** Doctor en Ciencias Administrativas.Profesor investigador de la Universidad Autónoma de Ciudad Juparez. Contacto: jesus.urrutia@uacj.mx. ORCID: O000-0003-2846-4811

20 Vol. 10, Núm. 19 Julio-Diciembre 2020 
problemas relacionados a la creación y comercialización del valor generado. Ante esta disyuntiva (hueco del conocimiento) surge la Teoría de las Partes Interesadas (Stakeholder Theory) como una forma de explicar la manera en que los diferentes agentes involucrados (Comunidades Locales, Turistas, Gobiernos Estatal y Federal), deben o participan en la planeación del desarrollo turístico de las comunidades anfitrionas y de los beneficios obtenidos y repartidos con base en su nivel de participación. Por lo cual se formula la siguiente pregunta ¿La teoría de las partes interesadas contribuye en las actividades correspondientes al turismo?

La teoría de las partes interesadas ha sido ampliamente aplicada a una diversidad de disciplinas, entre otras, cuidado de la salud, ética, administración y planificación turística, derecho, por mencionar algunas. (Parmar B., Freeman E., Harrison J., Wicks A., Colle S., 2010: 3-5).

\section{Revisión de Literatura}

El desarrollo del turismo, especialmente la formulación de políticas y la planeación, ha aceptado a la teoría de las partes interesadas porque el desarrollo del turismo ha estado acompañado de diversos grupos de involucrados con diferentes intereses e ideas sobre el costo y el beneficio del desarrollo. Un nuevo enfoque para resolver estos problemas ha sido perseguido, y se sugiere que todas las partes interesadas o afectadas por el turismo las actividades dentro de un mercado o comunidad particular, deben gestionar colectivamente el sistema de turismo (Kim, 2013: 59).

Del mismo modo, Sautter y Leisen (1999) argumentaron que los planificadores turísticos deberían tener una apreciación completa de todas las partes interesadas que tienen intereses en la planeación, el proceso, la entrega y los resultados de los servicios turísticos. Como señala Getz (1991), las perspectivas de los interesados en el desarrollo del turismo son fundamentales, lo que refuerza su actitud hacia el desarrollo. Estas perspectivas de los interesados varían porque los interesados tienen diferentes valores con respecto a los asuntos en los que están involucrados.

Por lo tanto, diferentes partes interesadas tienden a tener diversos valores que tienen un efecto en sus perspectivas sobre temas de desarrollo, y los planificadores de turismo deberían considerar los intereses de todos los interesados antes de proceder con los esfuerzos de desarrollo (Sautter y Leisen, 1999).

En particular, la identificación e involucramiento de las partes interesadas es el paso principal hacia el logro de asociaciones comunitarias y colaboración dentro del turismo (Hardy y Beeton, 2001). Citado por (Kim, 2013: 59)

La aplicación de los conceptos de la teoría de las partes interesadas al turismo requeriría planificadores del turismo darse cuenta y preocuparse por las perspectivas de diversos grupos de partes interesadas que participan en el sistema de turismo (Sautter y Leisen, 1999).

Freeman (1984) define "stakeholder" como "cualquier grupo o individuo que puede afectar o ser afectado por el logro de los objetivos de la empresa". En esta obra Freeman proporciona una guía $\mathrm{u}$ orientación para la gestión empresarial que permite comprender mejor las exigencias del entorno.

La teoría de los stakeholders supone es una perspectiva empresarial en la cual la maximización del beneficio del accionista deja paso a una administración fundamentada en la relación con los grupos de interés involucrados en las actividades turísticas. Por su parte, el compromiso de la empresa es hacia todos y cada uno de los implicados considerándolos en el momento de tomar las decisiones.

La teoría de las partes interesadas, iniciada por Freeman (1984), sugiere que una organización se caracteriza por sus relaciones con diversos grupos e individuos, incluidos empleados, clientes, proveedores, gobiernos y miembros de las comunidades. Según Freeman (1984: 46), la teoría de partes interesadas en una organización es (por definición) cualquier grupo o individuo que puede afectar o verse afectado por el logro de los objetivos de la organización.

Esto tiene que ver con cómo los clientes, proveedores, empleados, financieros, comunidades y gerentes se interrelacionan para crear y negociar el valor de manera conjunta. Entender el funcionamiento de la creación y negociar el valor es comprender cómo funcionan y cambian estas relaciones a lo largo del tiempo. El trabajo gerencial es administrar y dar forma a estas relaciones para crear el mayor valor posible para las partes interesadas y gestionar la distribución de ese valor (Freeman, 1984).

Esta teoría destaca el compromiso de los gerentes con la función de administración, elegir actividades para obtener beneficios óptimos para todos las partes interesadas identificadas. Se debe considerar a las partes interesadas, independientemente de su poder relativo o el interés de cada uno. La gerencia debe indagar de manera anticipada activamente las contribuciones de todas las partes interesadas, pues algunos pueden tener más fuerza que otros y esto no debería determinar la prioridad de la atención de la administración. Bajo esta teoría, todo el propósito de la empresa se convierte en la coordinación de los intereses de las partes interesadas.

Esta teoría admite que los gerentes actúan como si todos los diferentes intereses tuvieran un valor intrínseco, el reconocimiento de este valor y las obligaciones resultantes para el interesado le otorgan a la teoría su núcleo normativo. Esto no excluye el poder descriptivo o instrumental de la teoría. De hecho, los investigadores argumentan que su valor gerencial general proviene del hecho de que lo normativo, descriptivo, y los aspectos instrumentales de la teoría se apoyan mutuamente. Podría decirse que, sin embargo, el papel dominante de las teorías es su capacidad de proporcionar pautas 
morales y filosóficas para la gestión de una organización (Donaldson y Preston 1995).

Como tal, una gestión eficaz exige una atención sincrónica a los intereses genuinos de todos los interesados apropiados (Donaldson y Preston 1995). Clarkson (1995) enfatiza esta premisa y advierte que la falta de retención de la participación de incluso un único grupo de partes interesadas primarias dará como resultado la falla de la organización. Una organización que maneja eficazmente a sus partes interesadas debe comprender tres conceptos clave: la identificación del interesado y sus respectivas apuestas percibidas, los procesos necesarios para administrar.

Para Donaldson y Preston (1995: 67) la organización es entendida como una coordinación de intereses de las partes interesadas y la entidad de optimización requiere que la empresa acepte dos conceptos claves: Primero, los interesados son personas o grupos con intereses legítimos en los aspectos de procedimiento y/o sustantivos de la actividad empresarial. Los interesados se identifican por sus intereses en la corporación, independientemente de si la corporación tiene algún interés funcional correspondiente en ellos. Segundo, los intereses de todos los interesados son de valor intrínseco. Es decir, cada grupo de partes interesadas merece consideración por sí mismo y no simplemente por su capacidad para promover los intereses de algún otro grupo, como el accionista.

Para Sautter y Leisen (1999: 313) la teoría de las partes interesadas tiene que ver con los involucrados (comunidades, turistas, anfitriones) en el desarrollo de las actividades turísticas y cómo se distribuyen los beneficios. La teoría de las partes interesadas tiene que ver con un enfoque colaborativo hacia el desarrollo. En resumen, una organización con Capacidades de Gestión de Partes Interesadas tiene procesos organizacionales para tomar estos grupos y sus participaciones en cuenta rutinariamente como parte de los procedimientos operativos estándar de la organización y que implementa un conjunto de transacciones u ofertas para equilibrar los intereses de estas partes interesadas para lograr el propósito de la organización.

En el caso de Jamal y Getz (1995: 188) definen estos esfuerzos de colaboración como "un proceso de toma de decisiones conjunta entre actores clave autónomos de un dominio de turismo comunitario interorganizacional [diseñado] para resolver problemas de planificación del dominio y/o para gestionar cuestiones relacionadas con el planificación y desarrollo del dominio".

La teoría de las partes interesadas se ha utilizado ampliamente en el turismo como interdependencia y su impacto en el proceso de desarrollo del destino turístico. La experiencia y conocimientos de las partes interesadas en la gestión del turismo y la participación de la comunidad en el largo plazo. También se ha observado que los conocimientos y la experiencia de las partes interesadas en la gestión del turismo, la colaboración en la planificación y el desarrollo del turismo los procesos y la colaboración comunitaria a largo plazo han tenido un papel significativo en la gestión de los destinos turísticos.

Las comunidades están más preocupadas por la incidencia del turismo, calidad de vida y el aspecto sostenibilidad de sus comunidades. Por su parte, las empresas turísticas atienden los aspectos que impactan a sus empresas tales como, su producto comercialización y atender la satisfacción plena de su producto, por su parte, los turistas enfatizan sus inquietudes de la calidad de los productos turísticos en destinos. (Dabphet, 2012).

La teoría de las partes interesadas, en donde se observa que tiene que ver con la manera en que los individuos o grupos involucrados (residentes, trabajadores, sector público y privado, turistas Organizaciones No Gubernamentales, etc.) en las actividades turísticas se ven afectadas por conseguir los objetivos. Esta Teoría ha sido aceptada con agrado en la planeación turística. Considerar los diferentes intereses de las partes involucradas es fundamental para contribuir a que la planificación turística sea eficiente y eficaz.

La teoría de los stakeholders puede ser un puente o nexo entre lateoría a la práctica de una ética organizacional. En este sentido, esta teoría permite pensar a la organización desde una concepción plural, es decir que está conformada por diversidad de partes involucradas, que se relacionan entre si, no sólo desde el punto de vista jurídico, sino que también establecen contratos morales, en donde se establecen formas de hacer basadas en valores compartidos. Justamente en la relación entre las partes involucradas en donde se conocerán y pondrán en común los valores que les permitirán satisfacer mutuamente las expectativas. (Saiz V., 2012: 7-8)

Dentro de esta teoría de las partes interesadas cuando éstas se encuentran en problemas, el gerente tendría que analizar los problemas desde la perspectiva de las necesidades de un grupo o partes interesadas en un ámbito más amplio en función a esta actividad la creación de valor será mayor para cada una de las partes. En cuanto a las concesiones los gerentes deberán analizar la manera de cómo realizarlas y llegar a mejorar las compensaciones para todas las partes interesadas.

\section{Marco Metodológico}

Para el desarrollo de la investigación del presente documento se utilizaron las siguientes palabras claves: "Stakeholder Theory", turismo y partes interesadas, haciendo uso de artículos en idioma español e inglés, en formato electrónico basados en revistas especializadas en turismo con alto factor de impacto. Los artículos fueron elegidos por su contenido y que abordaban en el título la variable de estudio seleccionada, posteriormente se fueron analizando 
cada uno de ellos y al no contener la variable dentro de los objetivos, se fueron reemplazando por otros que si contaran con los datos necesarios para que fueran funcionales para el estudio. Sin embargo, en la búsqueda se cuenta con un buen número de artículos que pertenecen a revistas con factor de impacto.

La principal característica para la selección de los artículos fue que debían contener la variable de stakeholders, turismo y teoría de stakeholders se realizó la búsqueda de artículos, de los cuales se fueron eliminando por no contener la característica de la variable y posteriormente por no contener la variable dentro de los objetivos.

Se llevó acabo la búsqueda en diversas bases de datos, algunos artículos de revistas indexadas, una de las bases de datos en donde se encontró mayor información fue Science Direct, y la forma de obtención fue a través de la biblioteca virtual de la Universidad Autónoma de Ciudad Juárez.

El perido de búsqueda de las variables se realizó en un tiempo de tres meses abarcando los meses de Julio - Octubre del 2019.

\section{Conclusiones}

Uno de los aspectos relevantes dentro de la teoría de las partes interesadas es que la toma de decisiones tiene que ser de manera conjunta involucrando a cada una las partes interesadas con la finalidad de solucionar los conflictos y promover perspectivas compartidas. La cooperación es una de las etapas en el proceso colaborativo. Aunque los beneficios de la cooperación son muchos, e incluyen la integración y la eficiencia en los recursos económicos en el proceso de planificación y la eliminación de la superposición de servicios, la cooperación por sí sola no resuelve el problema de la naturaleza fragmentada del desarrollo del turismo.

Un paso importante dentro de la teoría de las partes interesadas es el reunir a los diferentes interesados e intereses en la primera etapa en el establecimiento de procesos colaborativos efectivos. Estos procesos pueden incrementar la cooperación o colaboración entre las diversas partes interesadas para apoyar las metas y los objetivos de las organizaciones de turismo, y generar una base para responder de manera más efectiva a los cambios.

Se podría señalar que la participación de la comunidad en el desarrollo del turismo se puede considerar como la contribución de las comunidades locales en la toma de decisiones. Así también es necesario que en la planeación de las comunidades locales se beneficien económicamente del desarrollo del turismo. Comúnmente, la participación de la comunidad en el turismo en países como el nuestro se ha inclinado hacia el turismo, por lo que se tendría que hacer participar en los beneficios económicos a las comunidades locales.

\section{Referencias}

Clarkson, M. (1995). A Stakeholder Framework for Analyzing and Evaluating Corporate Social Performance. Academy of Management Review.

Dabphet S. (2012). The key stakeholders in the implementation of sustainable tourism development in two rural towns of Thailand. Department of Tourism. Faulty of Business, Economics and Communications. Naresuan University, Thailand

Donaldson T., and Preston L. E. (1995). The Stakeholder Theory of the Corporation: Concepts, Evidence, and Implications. Academy of Management Review.

Freeman, R. E. (1984). Strategic Management: A Stakeholder Approach. Boston: Pitman.

Getz. D. (1991) Festivals, Special Events and Tourism. Reinhold, NY: Van Nostrand.

Kim, K. B. (2013) "The perceived role of key stakeholders" involvement in sustainable tourism development. PhD thesis. University of Nottingham.

Jamal Tazim B., Getz Donald. (1995). Collaboration theory and community tourism

planning. Annals of Tourism Research. Elsevier Science.

Saiz, V. (2012). Teoría de los Stakeholders, una herramienta que facilita una gestión ética de la responsabilidad social empresaria. Aspectos antropológicos y éticos de la administración.

Sautter, E., Leisen, B. (1999) Managing stakeholders a Tourism Planning Model. Annals of Tourism Research.

Parmar B., Freeman E., Harrison J., Wicks A., Colle S., (2010). Stakeholder Theory: The State of The Art. Cambridge, U.K. Cambridge University Press. 